\title{
Paternal effect on embryo quality in diabetic mice is related to poor sperm quality and associated with decreased glucose transporter expression
}

\author{
Sung Tae Kim and Kelle H Moley \\ Department of Obstetrics and Gynecology, Washington University in St Louis, 660 South Euclid Avenue, St Louis, \\ Missouri 63110, USA \\ Correspondence should be addressed to KH Moley; Email: moleyk@wustl.edu
}

\begin{abstract}
The objective of this study was to determine whether sperm quality, fertilization capacity, and subsequent embryo development are altered in diabetic male mice and whether differences in facilitative glucose transporter (GLUT; now known as solute carrier family 2, SLC2A) expression in the testis and sperm exist. Using two type 1 diabetic mouse models, SLC2A expression in the testis and sperm was determined by western immunoblotting and immunofluorescence staining. To address sperm quality and fertilization capacity, computer-assisted sperm analysis and in vitro fertilization were performed. SLC2A1, SLC2A3, and SLC2A5 did not change in expression in the testes or sperm between diabetic and non-diabetic mice. SLC2A8 and SLC2A9b were less expressed in the testes of both diabetic models versus controls. SLC2A9a was not

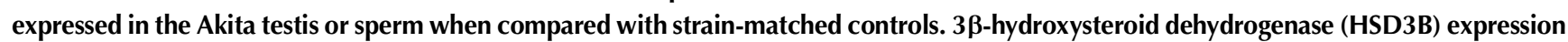
was significantly decreased in the Leydig cells from the diabetic mice. Sperm concentration and motility were significantly lower in both the diabetics when compared with the control. These parameters normalized in Akita diabetic males treated with insulin. In addition, fertilization rates were significantly lower in the Akita group (17.9\%) and the streptozotocin (STZ)-injected male group (43.6\%) when compared with the normal group (88.8\%). Interestingly, of the fertilized zygotes, embryo developmental rates to the blastocyst stage were lower in both diabetic models $(7.1 \%$ Akita and $50.0 \%$ STZ) when compared with controls $\mathbf{( 7 1 . 7 \% ) . ~ M a l e ~ d i a b e t e s ~ m a y ~ c a u s e ~ m a l e ~ s u b f e r t i l i t y ~ b y ~ a l t e r i n g ~}$ steroidogenesis, sperm motility, and SLC2A expression. This is the first study to link a paternal metabolic abnormality to a sperm effect on cell division and subsequent embryonic development.

Reproduction (2008) 136 313-322
\end{abstract}

\section{Introduction}

Diabetes has been associated with reproductive impairments in both men and women. Male reproductive alterations have been widely reported in individuals with diabetes. Many studies have documented abnormalities in testicular function and spermatogenesis in diabetic animals (Murray et al. 1983, Seethalakshmi et al. 1987, Scarano et al. 2006). In men affected by insulin-dependent diabetes, sperm have severe structural defects (Baccetti et al. 2002), significantly lower motility, and lower ability to penetrate hamster eggs (Shrivastav et al. 1989). The administration of high doses of streptozotocin (STZ) to male rodents induces a decrease in testicular testosterone production (Sanguinetti et al. 1995, Scarano et al. 2006).

Glucose is an important energy substrate for most mammalian cells. The entry of glucose into the cells is facilitated by a family of glucose transporters (GLUTs; now known as solute carrier family 2, SLC2A) that are characterized by the presence of 12 membrane-spanning helices and several conserved sequence motifs. Currently, there are 13 members of the facilitative glucose transporter family: SLC2A1-12 and SLC2A13, which was previously known as the $\mathrm{H}+$ coupled myo-inositol-transporter (HMIT; Joost et al. 2002, Wood \& Trayhurn 2003). The SLC2A family of proteins has been subdivided into three classes: class I consists of SLC2A1-4; class II consists of SLC2A5, SLC2A7, SLC2A9, and SLC2A11; and class III consists of SLC2A6, SLC2A8, SLC2A10, SLC2A12, and SLC2A13 (Joost \& Thorens 2001, Joost et al. 2002). SLC2As exhibit a high degree of sequence homology; however, they differ in their substrate specificity, kinetic characteristics, and tissue and subcellular distribution, and their response to extracellular stimuli (Joost et al. 2002, Riley et al. 2006).

SLC2A8 is a recently cloned member of the class III facilitative transporters and is expressed in the blastocyst stage embryo, heart, skeletal muscle, brain, spleen, prostate, intestine, and testis (Carayannopoulos et al. 2000, Doege et al. 2000, Gomez et al. 2006). Currently, we have confirmed that SLC2A8 is highly expressed in the Leydig cells as well as in the innermost cells of the seminiferous tubules in the mouse testis and localizes in the acrosome, midpiece, and principal piece of the mouse 
sperm (Kim \& Moley 2007). Human SLC2A9 was originally cloned from human kidney cDNA (Phay et al. 2000) and we have identified and cloned mouse SLC2A9 (Carayannopoulos et al. 2004). Sequence analysis has shown that SLC2A9 is most similar to SLC2A11, and both are categorized to the class II subgroup that also includes SLC2A5 and SLC2A7. A unique feature of mSLC2A9 is the existence of two predominant splice variants: $\mathrm{mSLC} 2 \mathrm{~A} 9 \mathrm{a}$ and mSLC2A9b differing only at the N-terminal intracytoplasmic tail (Keembiyehetty et al. 2006). We have shown that SLC2A9a and SLC2A9b are expressed in the mouse testis including the Leydig cells (Kim \& Moley 2007). SLC2A9a strictly localizes in the midpiece, but SLC2A9b localizes in the acrosome, midpiece, and principal piece of the mouse sperm (Kim \& Moley 2007). We have shown that surface SLC2A8 expression is regulated by the insulin/ insulin-like growth factor-1 (IGF1) signaling pathways through the IGF1 receptor in the mouse blastocyst (Carayannopoulos et al. 2000, Pinto et al. 2002). In different diabetic models, hepatic SLC2A8 has shown altered expression patterns (Gorovits et al. 2003). We have also reported that SLC2A9 protein expression was significantly increased in the kidney and liver from STZinduced diabetic mice compared with non-diabetic mice (Keembiyehetty et al. 2006). Although SLC2A8 (Ibberson et al. 2002, Schurmann et al. 2002, Chen et al. 2003, Gomez et al. 2006, Kim \& Moley 2007) and SLC2A9 (Kim \& Moley 2007) are expressed in the testis and sperm, it is not known whether their expression or localization is altered in the diabetic male reproductive system or not. The objective of this study was to determine whether sperm quality, fertilization capacity, and subsequent embryo development are altered in diabetic male mice and whether differences in facilitative glucose transporter (SLC2A) expression in the testis and sperm exist.

\section{Results \\ Expression of SLC2A8, SLC2A9a, and SLC2A9b is altered in diabetic testis and sperm}

In order to detect changes in the expression and localization of SLC2A1, SLC2A3, SLC2A5, SLC2A8, SLC2A9a, and SLC2A9b in the diabetic testis, RT-PCR as well as immunofluorescence staining and western blot analysis were performed. No changes in SLC2A1, SLC2A3, or SLC2A 5 expression were detected (data not shown), and these findings were consistent with previous reports in diabetic rat (Burant \& Davidson 1994). SLC2A8 mainly localized in a punctated intracellular compartment of the normal testis (Fig. 1A, panel a). However, the overall expression of SLC2A8 protein was slightly decreased in the STZ-injected testis (Fig. 1A, panel b) and markedly decreased in the Akita testis (see box in Fig. 1A, panel c). SLC2A9a protein was similarly expressed in the normal and STZ-injected testis (Fig. 1A, panels d-e). However, SLC2A9a was not expressed in the Akita testis (Fig. 1A, panel f). As shown in Fig. $1 \mathrm{~B}$ and $\mathrm{C}$, we confirmed that SLC2A9a protein was not expressed in the Akita testis, although Slc2a9a mRNA was expressed. SLC2A9b was less expressed in both of the diabetic testes when compared with normal testes (Fig. 1A, panels g-i and 1C).

Next, we examined SLC2A localization in the diabetic sperm by indirect immunofluorescence staining. SLC2A8 similarly localized in midpiece and principal piece as well as the acrosomal region of the normal and both of the diabetic sperm (Fig. 2A, panels a-c). SLC2A9a localized in the midpiece of the normal and STZ-injected sperm (Fig. 2A, panels $\mathrm{d}-\mathrm{e}$ ), but SLC2A9a was not detected in the Akita sperm (Fig. 2A, panel f). As shown in Fig. 2B, we confirmed that SLC2A9a protein was not expressed in the Akita sperm similar to the lack of expression in the testis. SLC2A9b similarly localized in the acrosomal region, midpiece, and principal piece of the normal and diabetic sperm (Fig. 2A, panels g-i). SLC2A9b expression in the acrosome and principal piece was significantly lower in the Akita sperm than in the normal and STZ-injected sperm (see the arrows in Fig. 2A, panel i). We confirmed SLC2A protein expression in the sperm by western blot analysis (Fig. 2B).

\section{Insulin protein is unchanged in location but decreased in expression in diabetic sperm}

Insulin in the normal and STZ-injected sperm was similarly expressed and localized in the acrosomal region, midpiece, and principal piece (Fig. $3 \mathrm{~A}$ and $\mathrm{B}$ ), but insulin was significantly decreased in the Akita sperm (Fig. 3C). Because STZ injection destroys only the pancreas, STZ-injected sperm should still express insulin as much as normal sperm. Since the Akita mouse has a single amino acid substitution of the insulin 2 gene, Akita sperm might show faint staining with insulin.

\section{SLC2A8, SLC2A9a, and HSD3B expression are decreased in isolated diabetic Leydig cells}

As shown in Fig. 4, SLC2A8 protein was slightly lower in both of the diabetic Leydig cells than in the normal Leydig cells. SLC2A9a was also significantly decreased in STZinjected Leydig cells and was not detected in the Akita Leydig cells. 3 $\beta$-hydroxysteroid dehydrogenase (HSD3B), which catalyzes the conversion of 3 - $\beta$-hydroxy-5-ene steroids to 3-oxo-4-ene steroids (progesterone and androstenedione), was significantly decreased in both of the diabetic Leydig cells. This result provides supporting evidence as to why diabetic males' testosterone levels are lower than normal males' levels (Scarano et al. 2006).

\section{Sperm concentration, motility, and fertility were compromised in diabetic male mice}

To examine the quality and fertility of the diabetic sperm, computer-assisted sperm analysis (CASA) and in vitro 
A
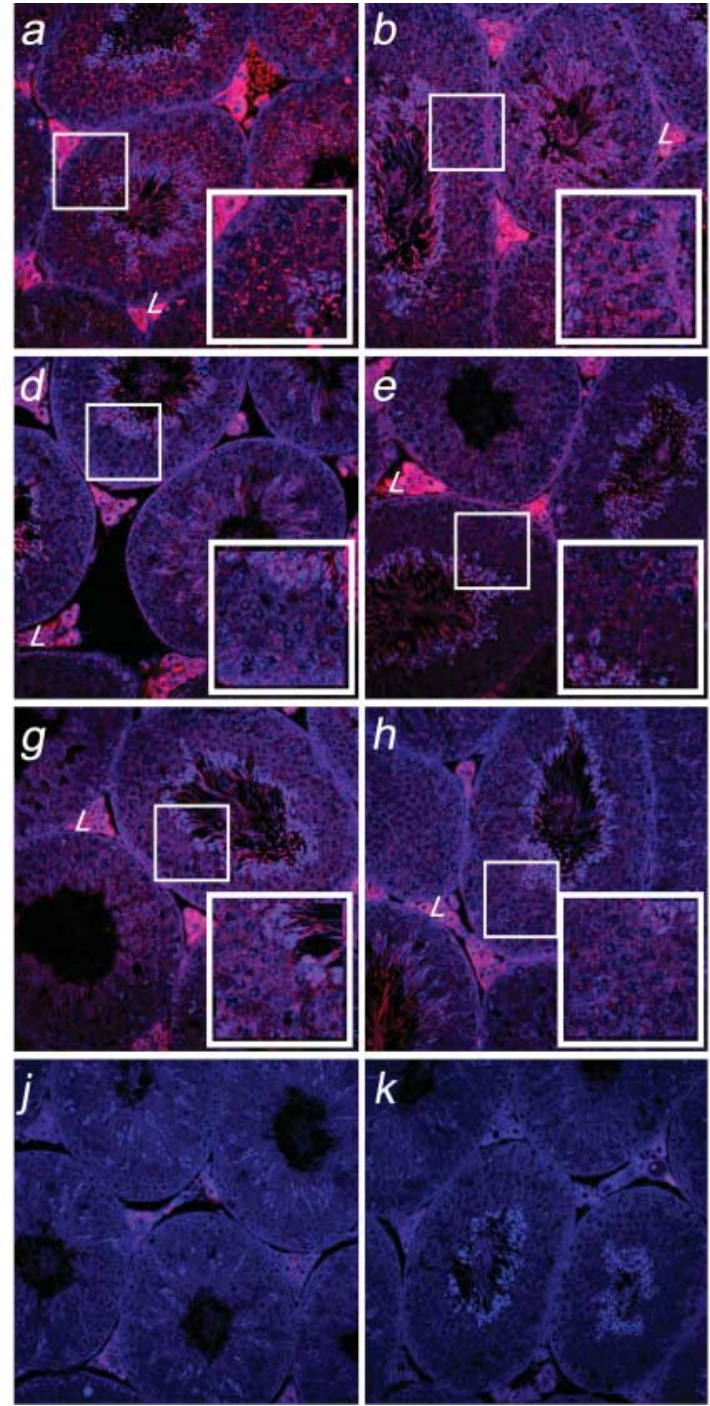
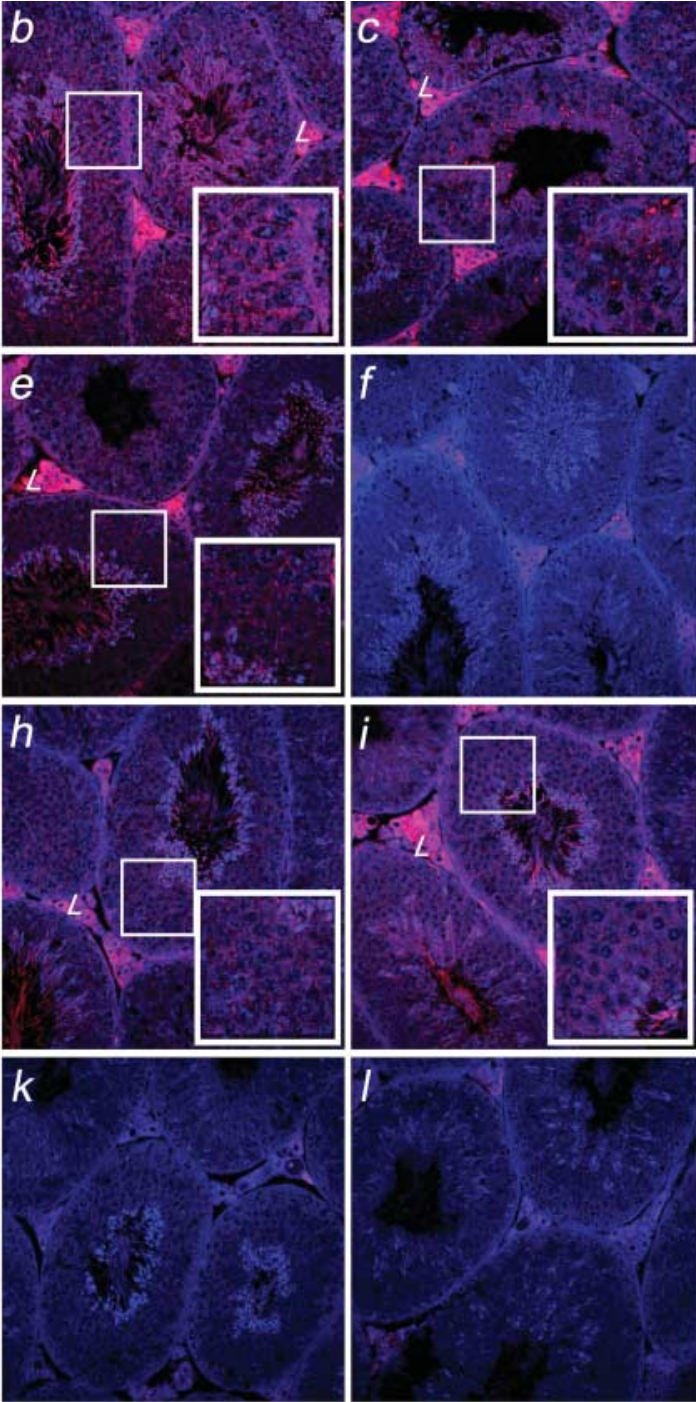

B

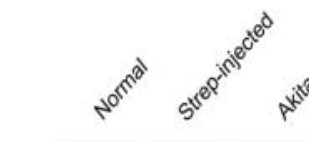

Slc2a8

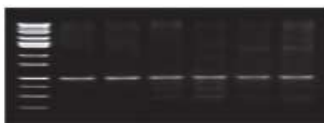

Slc2a9a

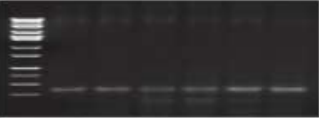

Slc2a9b

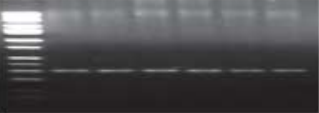

Gapdh

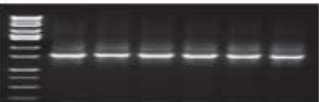

C

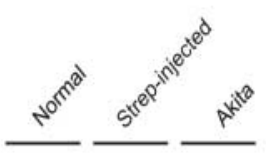

SLC2A8
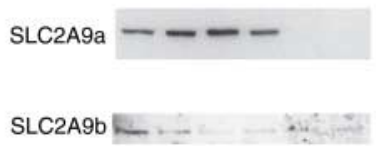

$\beta$-actin

Figure 1 (A) Immunofluorescence of SLC2A8 $(a-c)$, SLC2A9a $(d-f)$, and SLC2A9b (g-i) in the normal (a, d, g, and j), STZ-injected (b, e, h, and k), and Akita (c, f, i, and I) testis. (j, k, and I) Negative controls. Bouin's fixed paraffin-embedded testicular sections were incubated with anti-SLC2A8, antiSLC2A9a, or anti-SLC2A9b antibodies. The sections were then incubated with a secondary antibody, Alexa Fluor 546 goat anti-rabbit immunoglobulin G (red fluorescence). To-Pro-3 iodide was used to stain the nuclei (blue fluorescence). This experiment was performed over three times per group with at least five animals in each group for each experiment. Comparative RT-PCR (B) and western blot analysis (C) of SLC2A8, SLC2A9a, and SLC2A9b in the normal, STZ-injected, and Akita testis. Gapdh (B) and $\beta$-actin (C) were used as internal control for RT-PCR and western blot analysis respectively.

fertilization (IVF) were performed. Cauda epididymal sperm concentration was significantly lower in both types of the diabetic male mice than in normal mice (Fig. 5A, panel a). In the diabetic male mice, the percentage of motile sperm was significantly decreased (Fig. 5A, panel b) and the percentage of motile sperm with progressive motility was also decreased by 50\% (Fig. 5A, panel c). Path velocity, progressive velocity, curvilinear velocity, and lateral amplitude were all significantly reduced in the diabetic mice (data not shown). Beat frequency of both types of the diabetic males was same as that of the normal males. Straightness and linearity of Akita mice were slightly lower than those of STZ-injected and normal mice. The percentage of rapidly moving sperm was lower, and the percentage of static sperm was higher in both types of the diabetic males than in the normal males (Fig. 5A, panels $\mathrm{d}$ and e). Next, we performed IVF with normal cumulus-oocyte complex (COC) and diabetic sperm.

As shown in Fig. 5B and $\mathrm{C}$, the normal sperm group exhibited $88.8 \%$ of the fertilization rate, with $71.7 \%$ of fertilized embryos developing to the blastocyst stage. On the other hand, sperm isolated from STZ-injected mice showed a markedly decreased fertilization rate $(43.6 \%)$ with $50 \%$ of the fertilized embryos developing to the blastocyst stage. Sperm isolated from Akita mice also showed a significantly decreased fertilization rate $(17.9 \%)$ with $7.1 \%$ of the fertilized embryos developing to the blastocyst stage. In fact, control oocytes fertilized 

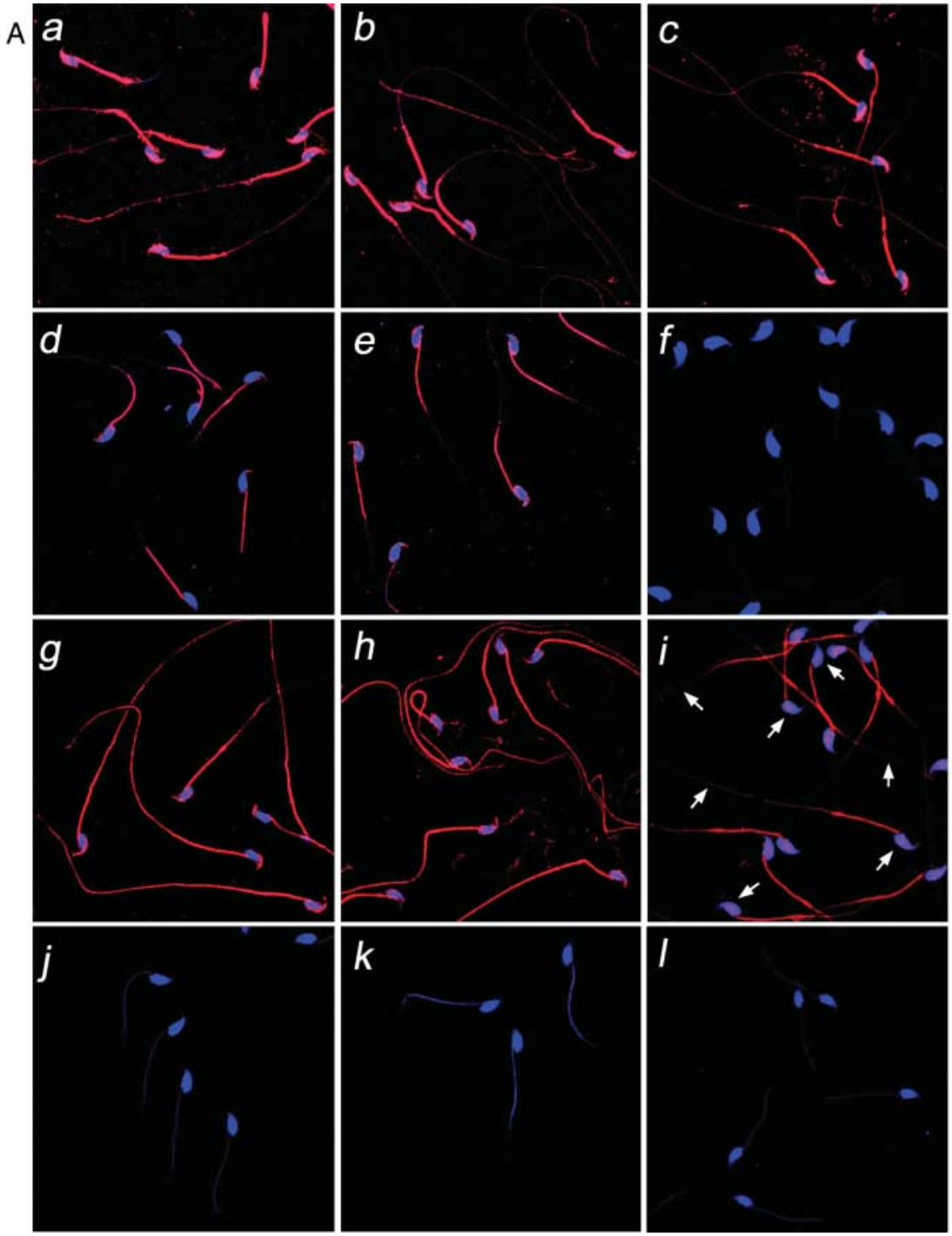
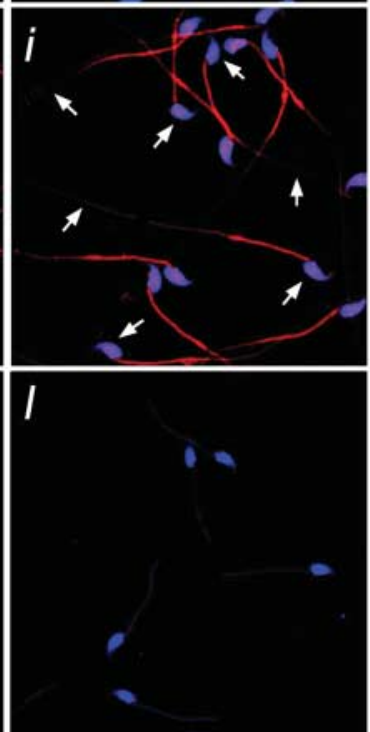

B

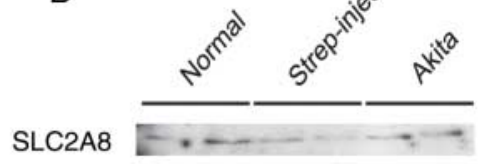

SLC2A9a - - - -

SLC2A9b

$\beta$-Actin

Figure 2 (A) Immunofluorescence of SLC2A8 (a-c), SLC2A9a (d-f), and SLC2A9b (g-i) in the normal (a, d, g, and j), STZ-injected (b, e, h, and k), and Akita $(\mathrm{C}, \mathrm{f}, \mathrm{i}$, and I) sperm. (j, $\mathrm{k}$, and I) Negative controls. Paraformaldehyde-fixed sperm were incubated with anti-SLC2A8, anti-SLC2A9a, or anti-SLC2A9b antibodies. The sperm were then incubated with a secondary antibody, Alexa Fluor 546 goat anti-rabbit immunoglobulin G (red fluorescence). To-Pro-3 iodide was used to stain the nuclei (blue fluorescence). This experiment was performed over three times per group with at least five animals in each group for each experiment. Arrows are directed at SLC2A9b expression at the acrosome and principal piece in the Akita sperm. (B) Western blot analysis of SLC2A8, SLC2A9a, and SLC2A9b in the normal, STZ-injected, and Akita sperm. $\beta$-actin was used as internal control.

with STZ-injected and Akita sperm showed increased fragmentation during cleavage stages and poor embryo developmental quality.

\section{Insulin treatment of Akita mice improves sperm motility and concentration}

Insulin- or placebo-containing pellets were placed in non-diabetic and diabetic Akita mice. Insulin pellet therapy for 7 days significantly increased the sperm total and progressive motility (51 and $103 \%$ increase respectively) as well as increasing total sperm concentration $(51 \%)$.

\section{Discussion}

It is well known that the fertility of germ cells is directly linked with glucose metabolism. Glucose is required specifically for sperm fusion to zona-free murine oocytes. Studies by Urner \& Sakkas (1996) have established that glucose must be transported into and metabolized by the male gamete in order for fertilization to occur. Moreover, glucose, not fructose or any other hexose sugars, is required in the medium during fusion for successful fertilization as well as to assure maintenance of viability of the embryo throughout the preimplantation period (Sakkas et al. 1993). In addition, spermatogenesis is 

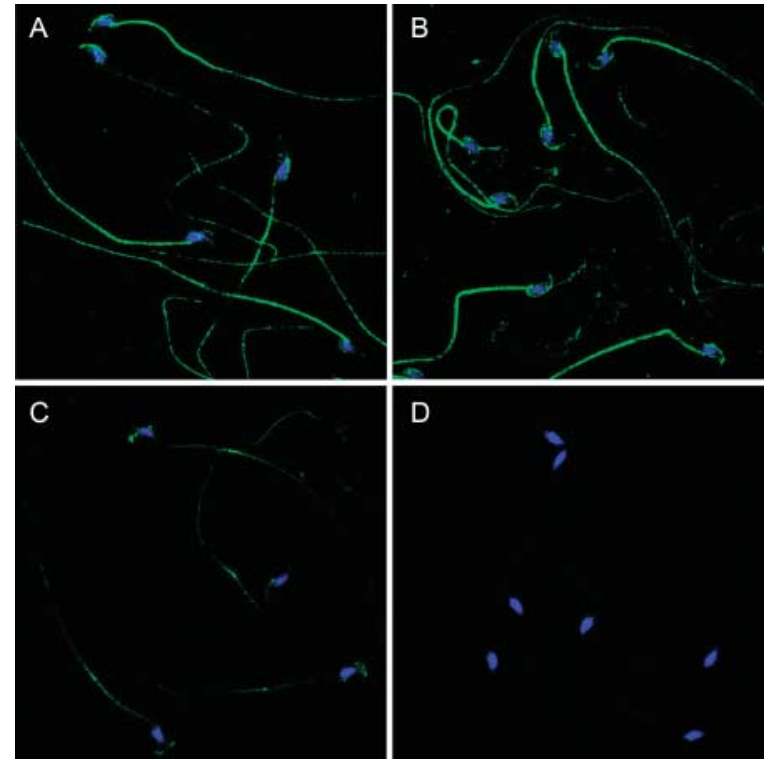

Figure 3 (A) Immunofluorescence of insulin in the normal, (B) STZinjected, and (C) Akita sperm. (D) Negative control. Paraformaldehydefixed sperm were incubated with anti-insulin antibody. The sperm were then incubated with a secondary antibody, Alexa Fluor 488 goat antiguinea pig immunoglobulin G (green fluorescence). To-Pro-3 iodide was used to stain the nuclei (blue fluorescence). This experiment was performed over three times per group with at least five mice per group for each experiment.

disturbed in diabetes and responsible in part for infertility (Sexton \& Jarow 1997, Glenn et al. 2003). However, factors involved in the development of male infertility with insulin-dependent diabetes are poorly understood. SLC2A8 is highly expressed in the testis

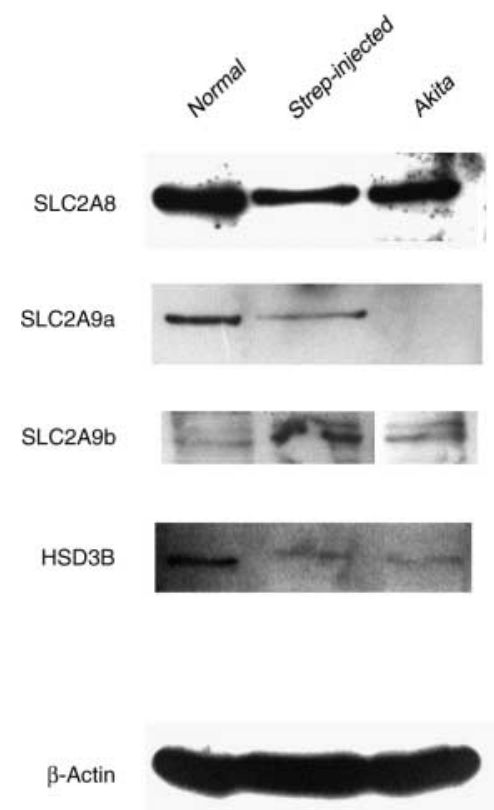

Figure 4 Western blot analysis of SLC2A8, SLC2A9a, SLC2A9b, and HSD3B in the Leydig cells from the normal, STZ-injected, and Akita testis. $\beta$-actin was used as internal control.
(Carayannopoulos et al. 2000, Doege et al. 2000, Gomez et al. 2006, Kim \& Moley 2007) and as such may play a major role in substrate delivery to develop sperm. These observations led us to focus on SLC2A expression in diabetic male mice. The most commonly used animal models of diabetes include rodents, dogs, and primates induced by chemical toxins such as STZ or alloxan. However, toxin-induced diabetes in mice has been less successful because of strain-dependent resistance to STZ (Rossini et al. 1977). We have used two different kinds of type 1 diabetic models, STZ-induced and Akita mice. By western immunoblotting and immunofluorescent microscopy, the total amount of SLC2A8 protein was decreased in the testis, Leydig cells, and sperm of both diabetic models. Furthermore, the plasma membrane localization of SLC2A8 was slightly decreased in the STZinjected testis and markedly decreased in the Akita testis. Recently, we have shown that SLC2A9a and SLC2A9b are expressed in the mouse testis and differentially localize in the sperm (Kim \& Moley 2007). As shown in Figs 1 and 2, SLC2A9a protein was not expressed in the Akita testis and sperm, although Slc2a mRNA was expressed. This absence of SLC2A9a protein expression in the Akita mice may result from some post-transcriptional events regulated by insulin signaling, which have been described previously (Freeman \& Wolf 1994, Han et al. 1995). SLC2A9b expression in the acrosome and principal piece was significantly lower in the Akita sperm than in the normal and STZ-injected sperm. The present study indicates that expression of SLC2A9 as well as SLC2A8 may be regulated by insulin signaling and/or hyperglycemia and may play an important role in sperm maturation and fertilization. Overall, diabetes might lead to the alteration of SLC2A expression in the sperm and testis.

These conclusions are consistent with our prior studies in rodent preimplantation embryos as well as other studies in human, rodent, and ovine placental tissue showing decreased SLC2A expression in models of maternal diabetes (Das et al. 1998, Hahn et al. 1998, Moley et al. 1998b, Chi et al. 2000a, Eng et al. 2007). Maternal high glucose concentrations lead to subsequent downregulation of SLC2A expression and result in decreased intracellular glucose, triggering a cell death pathway which we have shown is dependent on BAX and p53 expression (Moley et al. 1998a, Keim et al. 2001). Recent work has shown that exposing preimplantation embryos to type 2 diabetic conditions in vitro, specifically hyperinsulinemia and hyperglycemia, leads to apoptosis, decreased glucose uptake and decreased expression of SLC2A8 protein in the blastocyst stage (Chi et al. 2000b, Eng et al. 2007). This work now suggests that male gametes respond in a similar fashion with a change in the expression of SLC2As in response to paternal diabetes.

According to recent reports, insulin is expressed in human ejaculated sperm (Aquila et al. 2005) and epididymal mouse sperm (Kim \& Moley 2007), and it 

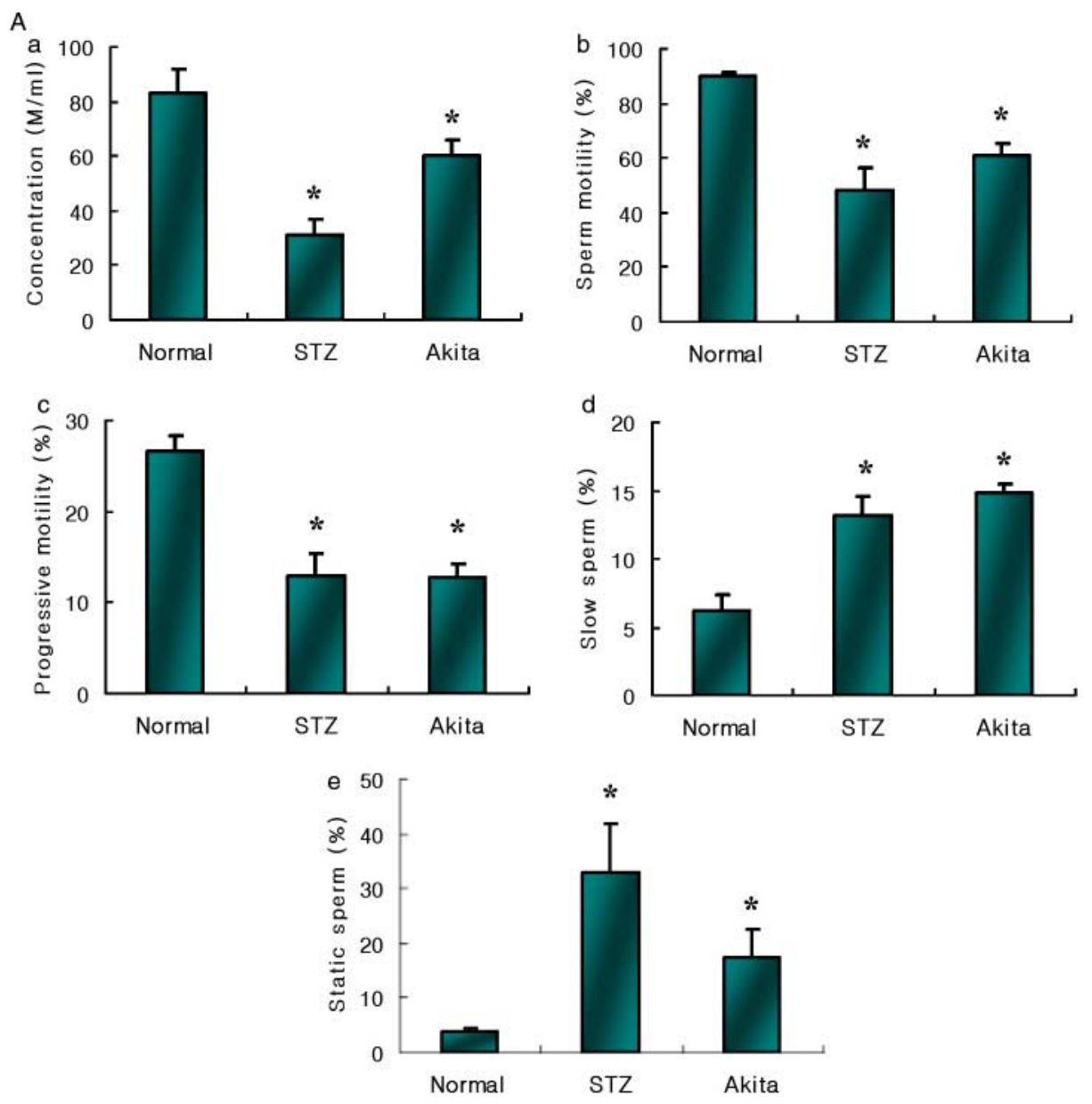

B

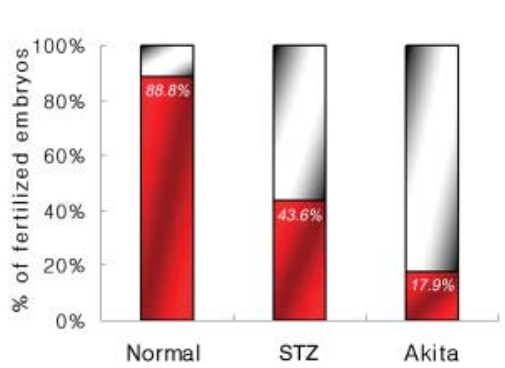

C

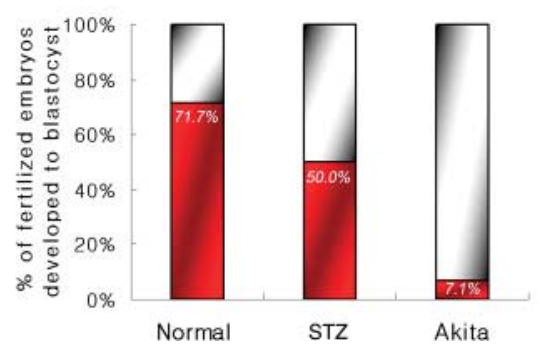

Figure 5 Computer-assisted sperm analysis (CASA) and in vitro fertilization (IVF). (A) Mean sperm concentration: (a) motility, (b) progressive motility, (c) slow, (d) and static (e) motility in normal, STZ-injected, and Akita males. Asterisks indicate significant differences in normal group. (B) Percentage of fertilized embryos with normal oocytes. (C) Percentage of fertilized embryos developed to blastocyst. might provide an autocrine regulation of glucose metabolism by sperm (Aquila et al. 2005). In this study, we confirmed these findings by detecting insulin in control and STZ-injected diabetic mouse as shown in Fig. $3 \mathrm{~A}$ and $\mathrm{B}$. But insulin detection in the sperm from Akita diabetic mice is fainter than that of the normal and STZ-injected sperm. These mice have a single amino acid substitution in the insulin 2 gene causing misfolding of the insulin protein (Barber et al. 2005). Insulin is detected in secretory granules in Akita mice; however, it is profoundly decreased in concentration, as we showed here (Wang et al. 1999). Interestingly, treating the Akita mice with insulin normalized the sperm concentration, and total and progressive motility of the mice, suggesting that insulin signaling and increased SLC2A9 expression improves sperm quality in diabetic mice.

To determine sperm quality and fertilization capacity of sperm from diabetic versus control mice, we performed CASA and IVF with normal COC and cauda epididymal sperm. The epididymis imparts sperm maturation by providing a specific fluid environment of secretory products from its epithelium and serves as a site for the sperm to acquire motility and fertilizing potential (Amann et al. 1993, Cooper 1998). In addition to sperm maturation, the epididymis plays a significant role in the transport, concentration, protection, and 
storage of the sperm. Thus, the inevitable role of the epididymis in sperm maturation and the adverse effects of diabetes on testicular functions are well established. However, there is not enough information on the impact of diabetes on the epididymis. As shown in Fig. 5, this study supports, in part, that diabetes could lead to reduced sperm motility and fertility as well as compromised epididymal functions for sperm maturation. The sperm isolated from STZ-injected and Akita males showed markedly low rates of fertilization and development to the blastocyst stage. Although litter size of STZinjected and Akita male groups through the natural mating with normal females was significantly lower than that of normal male group, in vivo fertilization rate and embryo development were higher than that of in vitro (Table 1). These differences probably reflect the different origin of the sperm. For IVF, we used cauda epididymal sperm that contains a much higher percentage of nonmotile sperm. In natural mating, motile and good quality sperm are naturally selected from the vas deferens and female reproductive tract during ejaculation and before fertilization.

Previous studies have also demonstrated paternal effects on cell division in human preimplantation embryos obtained by assisted reproductive technologies (Menezo 2006). It was first proposed by Renard \& Babinet (1986) that some factor in the male gamete acted at the pronucleus stage to affect later steps in embryonic development. They demonstrated that proper nucleoplasmic interaction was necessary to allow normal embryo development. The exact factor, however, was not clear. In the early 1990s, this paternal effect idea was brought into the clinical realm when Janny \& Menezo (1994) reported that the quality of sperm did determine developmental potential of IVF embryos, contrary to popular view. It has been speculated that embryo quality may be negatively affected by deficiencies in the sperm nuclear genome or sperm-derived cytoplasmic factors, specifically the centrosome as well as oocyte-activating factor, responsible for calcium homeostasis in the oocyte (Tesarik 2005). Early paternal effects can be detected as early as the one-cell zygote and are associated with poor zygote and embryo morphology and low cleavage speed. This pathology is not thought to associated with increased sperm DNA damage, but more likely this is due to as of yet unidentified cytoplasmic factors. Centrioles and oocyte activation factors have been raised, but it is still speculative. By contrast, one study

Table 1 Comparison of litter size.

\begin{tabular}{lcc}
\hline Male & Female & Litter size \\
\hline Normal & Normal & 9.3 \\
STZ injected & & $2.3^{*}$ \\
Akita & & $5.8^{*}$ \\
\hline
\end{tabular}

*Significantly differ from the normal group $(P<0.01)$. disputes this hypothesis and reports lower blastocyst formation rates in human ICSI procedures with DNA damaged sperm (Nasr-Esfahani et al. 2005). This study showed that DNA fragmentation as detected by the Comet assay does not preclude fertilization; however, the embryos derived from these sperm have a lower potential to reach the blastocyst stage. All these studies are done in ICSI male factor patients whose sperm parameters fall below normal World Health Organization values.

In this study, the diabetic mouse sperm parameters were also abnormal. Although the exact etiology of these abnormalities is not known, the growing evidence indicates that oxidative stress is increased in diabetes, due to the overproduction of reactive oxygen species (ROS) and decreased efficiency of antioxidant defenses (Giron et al. 1999, Wiernsperger 2003). Oxidation of lipids, proteins, and other macromolecules such as DNA occurs during the development of diabetes (Ohkawa et al. 1979), and mitochondrial DNA mutations have also been reported in diabetic tissues, suggesting oxidative stress-related mitochondrial damage (Lee et al. 1997). Mammalian sperm cells present a specific lipid composition with a high content of polyunsaturated fatty acids (plasmalogens and sphingomyelins). The lipids in spermatozoa are the main substrates for peroxidation and excess amounts of ROS and free radicals have adverse effects on sperm motility and fertility (Aitken et al. 1989). Furthermore, oxidative damage to lipids and DNA of spermatozoa is associated with declining motility and diminished fertility of human sperm (Chen et al. 1997, Kao et al. 1998). In this regard, decreased IVF rate in STZ-injected and Akita males might result from oxidative stress from dead sperm, but this stress should be more or less overcome through in vivo selection procedures during ejaculation and before fertilization. In fact, oocytes fertilized with STZ-injected and Akita sperm showed fragmentation and poor embryo development, which may be also affected by oxidative stress from sperm. The molecular mechanisms of reduced spermatogenesis, motility, and fertilization capacity in diabetes, however, remain to be elucidated.

In addition insulin treatment of the Akita mice for 7 days changed the sperm motility and concentration. It has been reported that IGF1 and insulin as well as IGF1 promote spermatogonial differentiation into primary spermatocytes by binding to the IGF1 receptor (Nakayama et al. 1999). It has been also shown that both the sperm plasma membrane and the acrosome represent cytological targets for insulin (Silvestroni et al. 1992, Kim \& Moley 2007). We confirm here that insulin is present in mature sperm from control and STZ-treated mice but not Akita males. All reports suggest that insulin signaling may be important for spermatogenesis, sperm maturation, and capacitation, and support our results suggesting that insulin-deficient diabetic mice show 
decreased sperm quality and fertilization rate. Interestingly, SLC2A9b expression was increased in the insulin-treated Akita mice and coincided with improved sperm parameters.

In conclusion, diabetes in two different mouse models results in male subfertility by altering spermatogenesis, steroidogenesis, and sperm maturation. Insulin signaling might play an important role in SLC2A8 and SLC2A9 expression during spermatogenesis, steroidogenesis, and sperm maturation. The distribution of SLC2As suggests that they may play different roles not only in internal glucose movement, but also in the traffic of sugars for the maturation and motility maintenance of the sperm during the capacitation and fertilization processes. The present study should provide a clue for the treatment of male infertility in patients with diabetes.

\section{Materials and Methods}

\section{Animals}

Mice were housed according to Institutional Animal Care and Use Committee and National Institutes of Health guidelines. The 8-week-old B6XSJL F1 and type 1 diabetic Akita mice, which have significant hyperglycemia resulting from a single amino acid substitution in the insulin 2 gene and are on a $\mathrm{C} 57$ black background, were purchased from Jackson Laboratories, Bar Harbor, ME, USA. To generate a chemically induced diabetic model, B6XSJL male mice received four to five injections of STZ (Sigma Chemical Co. Ltd) at a dose of $100 \mathrm{mg} / \mathrm{kg}$ (dissolved in sodium citrate buffer $\mathrm{pH}$ 4.4). Seven-day post-injection, a tail blood sample was measured for glucose concentrations via a Hemocue B glucose analyzer (Stockholm, Sweden). Mice with blood glucose levels $\geq 300 \mathrm{mg} / \mathrm{dl}$ were selected. After 1 month, the testes and sperm were isolated. The STZ-induced B6XSJL mice, the Akita mice, and the non-injected B6XSJL mice were all age matched. The Akita mouse is on a C57 black background and in order to use the B6XSJL as a control, we compared C57 black age-matched males to B6XSJL males and found no differences in the sperm or testes SLC2A expression (data not shown). There were also no differences in sperm parameters using CASA (data not shown). For this reason, we used age-matched B6XSJL non-diabetic mice as the control for both diabetic groups.

\section{Total RNA extraction and RT-PCR}

Total RNA was extracted using the RNeasy mini kit (Qiagen) according to the manufacturer's instruction. RT with oligo(dT) priming was performed to generate cDNAs from $1 \mu \mathrm{g}$ total RNA using Superscript II (Invitrogen) following the instruction provided by the manufacturer. DNA amplification was carried out with Taq DNA polymerase (Invitrogen) using the primers in Table 1. Amplified fragments were separated by electrophoresis on $2 \%$ agarose gels and visualized by ethidium bromide staining.

\section{Immunohistochemistry and western immunoblot analysis of testes}

Immunohistochemistry of SLC2As in the testes was performed in Bouin's fixed paraffin-embedded or frozen sections as described previously (Keembiyehetty et al. 2006, Kim \& Moley 2007). In brief, the testes were removed, fixed in Bouin's Solution overnight, and embedded in paraffin. Testicular sections were incubated with anti-SLC2A8, antiSLC2A9a, or anti-SLC2A9b antibodies. The sections were then incubated with a secondary antibody, Alexa Fluor 546 goat anti-rabbit immunoglobulin G. To-Pro-3 iodide was used to stain the nuclei. Western blot analysis was performed on the testes as described previously (Kim \& Moley 2007).

\section{Immunofluorescence and western immunoblot analysis of sperm}

Indirect immunofluorescence of sperm was performed as described previously (Kim \& Moley 2007). In brief, paraformaldehyde-fixed sperm were incubated with anti-SLC2A8, anti-SLC2A9a, or anti-SLC2A9b antibodies. Sperm were then incubated with a secondary antibody, Alexa Fluor 546 goat anti-rabbit immunoglobulin G. To-Pro-3 iodide was used to stain the nuclei. Western blot analysis was performed on the sperm as described previously (Kim \& Moley 2007).

\section{IVF}

\section{Preparation of spermatozoa}

The cauda epididymis from male was removed at 12.0-13.0 h post-hCG injection and quickly transferred to a culture dish containing $500 \mu \mathrm{l} \mathrm{HTF}$ medium (Specialty Media, Phillipsburg, NJ, USA), then transferred to another dish with $500 \mu \mathrm{l} \mathrm{HTF}$ medium. Some incisions were made in the cauda epididymis, and gentle squeezing using fine forceps allowed the spermatozoa to swim out. Capacitation was allowed to proceed for $1.5 \mathrm{~h}$ at $37^{\circ} \mathrm{C}$ in a $5 \% \mathrm{CO}_{2}$ incubator. The concentration of the spermatozoa was determined, and if necessary, adjusted to obtain similar sperm concentrations from normal, STZinjected, and Akita males.

\section{Preparation of oocytes}

Adult non-diabetic B6 female mice that were 7- to 8-weeks old were induced to superovulate by i.p. injection of $5 \mathrm{IU}$ pregnant mare's serum gonadotropin followed by 5 IU hCG $48 \mathrm{~h}$ later. Animals were killed 14.0-15.0 h post-hCG injection. Oviducts were collected in a $35 \mathrm{~mm}$ dish containing $2 \mathrm{ml} \mathrm{HTF}$ medium. The COCs were recovered by gentle dissection of the oviducts. COCs were transferred to a $200 \mu \mathrm{l}$ droplet of HTF medium covered with mineral oil.

\section{Insemination and fertilization}

Capacitated spermatozoa in the range $1 \times 10^{6}$ to $2 \times 10^{6} / \mathrm{ml}$ were added to each insemination droplet with the COCs and placed in an incubator for 4-6 h. Oocytes then were washed 
two or three times with $100 \mu$ l potassium simplex optimized medium (Specialty Media) under oil and finally transferred to $50 \mu \mathrm{l}$ droplets of the same medium. The dishes were incubated at $37{ }^{\circ} \mathrm{C}$ in $5 \% \mathrm{CO}_{2}$ in a humidified incubator. Fertilization was assessed by recording the number of pronucleus and 2-cell embryos 9-10 h and $24 \mathrm{~h}$ after fertilization respectively. Embryos were observed at $200 \times$ magnification under the inverted microscope. To assess the level of parthenogenesis, eggs were incubated under the same conditions but without sperm addition. This level was consistently $0 \%$.

\section{CASA}

The functional analysis of male reproduction was determined by measuring sperm motility of mice using the CASA system (Hamilton-Thorne Research, Beverly, MA, USA). The cauda epididymis was diced, and the sperm were allowed to disperse into the medium for $10 \mathrm{~min}$ at $37^{\circ} \mathrm{C}$. Among the motility parameters measured were the following: percent motility - the percent of motile sperm within the analysis field divided by the sum of the motile plus immotile sperm within the analysis field; path velocity (VAP) - the average velocity of the smoothed cell path, expressed in microns per second; progressive velocity (VSL) - the average velocity measured in a straight line from the beginning to the end of the track; curvilinear velocity $(\mathrm{VCL})$ - the sum of the incremental distances moved in each frame along the sampled path divided by the time taken for the sperm to cover the track; amplitude of lateral head (ALH) - the ALH displacement corresponds to the mean width of the head oscillation as the sperm swims; beat cross frequency - the frequency with which the sperm track crosses the sperm path; straightness (STR) - the departure of the cell path from a straight line; and linearity (LIN) - the departure of the cell track from a straight line.

\section{Placement of insulin pellets}

LinBitTM insulin implants (LinShin Canada Inc., Scarborough, Ontario, Canada) were subcutaneously inserted under the middorsal skin of Akita male mice during anesthesia. According to the manufacturer's instructions (http://www.linshincanada.com), we added two implants for the first $20 \mathrm{~g}$ in body weight and another implant for each additional $5 \mathrm{~g}$. The same number of blanks was placed for use as controls. At 2 days post-surgery, a tail blood sample was measured for glucose concentrations via a Hemocue B glucose analyzer. Blood glucose levels $\geq 100 \mathrm{mg} / \mathrm{dl}$ were selected. After 1 week, the testes were isolated.

\section{Declaration of interest}

The authors declare that there is no conflict of interest that could be perceived as prejudicing the impartiality of the research reported.

\section{Funding}

This work was funded by NIH RO1HD040390 (KHM) and a research grant from the American Diabetes Association (KHM).

\section{References}

Aitken RJ, Clarkson JS \& Fishel S 1989 Generation of reactive oxygen species, lipid peroxidation, and human sperm function. Biology of Reproduction 41 183-197.

Amann RP, Hammerstedt RH \& Veeramachaneni DN 1993 The epididymis and sperm maturation: a perspective. Reproduction, Fertility, and Development 5 361-381.

Aquila S, Gentile M, Middea E, Catalano S \& Ando S 2005 Autocrine regulation of insulin secretion in human ejaculated spermatozoa. Endocrinology 146 552-557.

Baccetti B, La Marca A, Piomboni P, Capitani S, Bruni E, Petraglia F \& De Leo V 2002 Insulin-dependent diabetes in men is associated with hypothalamo-pituitary derangement and with impairment in semen quality. Human Reproduction 17 2673-2677.

Barber AJ, Antonetti DA, Kern TS, Reiter CE, Soans RS, Krady JK, Levison SW, Gardner TW \& Bronson SK 2005 The Ins2Akita mouse as a model of early retinal complications in diabetes. Investigative Ophthalmology \& Visual Science 46 2210-2218.

Burant CF \& Davidson NO 1994 GLUT3 glucose transporter isoform in rat testis: localization, effect of diabetes mellitus, and comparison to human testis. American Journal of Physiology 267 R1488-R1495.

Carayannopoulos M, Chi M, Cui Y, Pingsterhaus J \& Moley K 2000 SLC2A8, a glucose transporter responsible for insulin-stimulated uptake in the blastocyst. PNAS 97 7313-7318.

Carayannopoulos MO, Schlein A, Wyman A, Chi M, Keembiyehetty C \& Moley KH 2004 SLC2A9 is differentially expressed and targeted in the preimplantation embryo. Endocrinology 145 1435-1443.

Chen CS, Chao HT, Pan RL \& Wei YH 1997 Maintenance of human sperm motility and prevention of oxidative damage through co-culture incubation. Andrologia 29 227-233.

Chen Y, Nagpal ML \& Lin T 2003 Expression and regulation of glucose transporter 8 in rat Leydig cells. Journal of Endocrinology 179 63-72.

Chi MM, Pingsterhaus J, Carayannopoulos M \& Moley KH 2000a Decreased glucose transporter expression triggers BAX-dependent apoptosis in the murine blastocyst. Journal of Biological Chemistry 275 40252-40257.

Chi MM, Schlein AL \& Moley KH 2000 $b$ High insulin-like growth factor 1 (IGF-1) and insulin concentrations trigger apoptosis in the mouse blastocyst via down-regulation of the IGF1 receptor. Endocrinology 141 4784-4792.

Cooper TG 1998 Interactions between epididymal secretions and spermatozoa. Journal of Reproduction and Fertility Supplement $\mathbf{5 3}$ 119-136.

Das UG, Sadiq HF, Soares MJ, Hay WW Jr \& Devaskar SU 1998 Timedependent physiological regulation of rodent and ovine placental glucose transporter (GLUT-1) protein. American Journal of Physiology 274 R339-R347.

Doege H, Schurmann A, Bahrenberg G, Brauers A \& Joost HG 2000 SLC2A8, a novel member of the sugar transport facilitator family with glucose transport activity. Journal of Biological Chemistry 275 16275-16280.

Eng GS, Sheridan RA, Wyman A, Chi MM-Y, Bibee KP, Jungheim ES \& Moley KH 2007 AMPK activation increases glucose uptake, decreases apoptosis and improves pregnancy outcome in embryos exposed to high IGF-1 concentrations. Diabetes 57 2228-2234.

Freeman JE \& Wolf CR 1994 Evidence against a role for serine 129 in determining murine cytochrome P450 Cyp2e-1 protein levels. Biochemistry 33 13963-13966.

Giron MD, Salto R, Gonzalez Y, Giron JA, Nieto N, Periago JL, Suarez MD \& Hortelano P 1999 Modulation of hepatic and intestinal glutathione S-transferases and other antioxidant enzymes by dietary lipids in streptozotocin diabetic rats. Chemosphere 38 3003-3013.

Glenn DR, McClure N \& Lewis SE 2003 The hidden impact of diabetes on male sexual dysfunction and fertility. Human Fertility 6 174-179.

Gomez O, Romero A, Terrado J \& Mesonero JE 2006 Differential expression of glucose transporter SLC2A8 during mouse spermatogenesis. Reproduction 131 63-70.

Gorovits N, Cui L, Busik JV, Ranalletta M, Hauguel de-Mouzon S \& Charron MJ 2003 Regulation of hepatic SLC2A8 expression in normal and diabetic models. Endocrinology 144 1703-1711. 
Hahn T, Barth S, Weiss U, Mosgoeller W \& Desoye G 1998 Sustained hyperglycemia in vitro down-regulates the GLUT1 glucose transport system of cultured human term placental trophoblast: a mechanism to protect fetal development? FASEB Journal 12 1221-1231.

Han XX, Handberg A, Petersen LN, Ploug T \& Galbo H 1995 Stability of GLUT-1 and GLUT-4 expression in perfused rat muscle stimulated by insulin and exercise. Journal of Applied Physiology 78 46-52.

Ibberson M, Riederer BM, Uldry M, Guhl B, Roth J \& Thorens B 2002 Immunolocalization of GLUTX1 in the testis and to specific brain areas and vasopressin-containing neurons. Endocrinology 143 276-284.

Janny L \& Menezo YJ 1994 Evidence for a strong paternal effect on human preimplantation embryo development and blastocyst formation. Molecular Reproduction and Development 38 36-42.

Joost HG \& Thorens B 2001 The extended GLUT-family of sugar/polyol transport facilitators: nomenclature, sequence characteristics, and potential function of its novel members (review). Molecular Membrane Biology 18 247-256.

Joost HG, Bell GI, Best JD, Birnbaum MJ, Charron MJ, Chen YT, Doege H, James DE, Lodish HF, Moley KH et al. 2002 Nomenclature of the GLUT/SLC2A family of sugar/polyol transport facilitators. American Journal of Physiology. Endocrinology and Metabolism 282 E974-E976.

Kao SH, Chao HT \& Wei YH 1998 Multiple deletions of mitochondrial DNA are associated with the decline of motility and fertility of human spermatozoa. Molecular Human Reproduction 4 657-666.

Keembiyehetty C, Augustin R, Carayannopoulos MO, Steer S, Manolescu A, Cheeseman CI \& Moley KH 2006 Mouse glucose transporter 9 splice variants are expressed in adult liver and kidney and are up-regulated in diabetes. Molecular Endocrinology 20 686-697.

Keim AL, Chi MM \& Moley KH 2001 Hyperglycemia-induced apoptotic cell death in the mouse blastocyst is dependent on expression of $\mathrm{p} 53$. Molecular Reproduction and Development 60 214-224.

Kim ST \& Moley KH 2007 Glucose transporter (GLUT) 8, SLC2A9a and $\mathrm{SLC2A9b}$ protein expression in the mouse testis and sperm. Reproductive Sciences 14 445-455.

Lee PD, Giudice LC, Conover CA \& Powell DR 1997 Insulin-like growth factor binding protein-1: recent findings and new directions. Proceedings of the Society for Experimental Biology and Medicine 216 319-357.

Menezo YJ 2006 Paternal and maternal factors in preimplantation embryogenesis: interaction with the biochemical environment. Reproductive Biomedicine Online 12 616-621.

Moley KH, Chi MM-Y, Knudson CM, Korsmeyer SJ \& Mueckler MM 1998a Hyperglycemia induces apoptosis in preimplantation embryos via cell death effector pathways. Nature Medicine 12 1421-1424.

Moley KH, Chi MM-Y \& Mueckler M 1998b Maternal hyperglycemia alters glucose transport and utilization in mouse preimplantation embryos. American Journal of Physiology 275 E38-E47.

Murray FT, Cameron DF \& Orth JM 1983 Gonadal dysfunction in the spontaneously diabetic BB rat. Metabolism 32 141-147.

Nakayama Y, Yamamoto T \& Abe SI 1999 IGF-I, IGF-II and insulin promote differentiation of spermatogonia to primary spermatocytes in organ culture of newt testes. International Journal of Developmental Biology 43 343-347.

Nasr-Esfahani MH, Salehi M, Razavi S, Anjomshoa M, Rozbahani S, Moulavi F \& Mardani M 2005 Effect of sperm DNA damage and sperm protamine deficiency on fertilization and embryo development postICSI. Reproductive Biomedicine Online 11 198-205.

Ohkawa H, Ohishi N \& Yagi K 1979 Assay for lipid peroxides in animal tissues by thiobarbituric acid reaction. Analytical Biochemistry 95 351-358.
Phay JE, Hussain HB \& Moley JF 2000 Cloning and expression analysis of a novel member of the facilitative glucose transporter family, SLC2A9 (SLC2A9). Genomics 66 217-220.

Pinto A, Carayannopoulos M, Hoehn A, Dowd L \& Moley K 2002 SLC2A8 expression and translocation are critical for murine blastocyst survival. Biology of Reproduction 66 1729-1733.

Renard JP \& Babinet C 1986 Identification of a paternal developmental effect on the cytoplasm of one-cell-stage mouse embryos. PNAS 83 6883-6886.

Riley JK, Carayannopoulos MO, Wyman AH, Chi M \& Moley KH 2006 Phosphatidylinositol 3-kinase activity is critical for glucose metabolism and embryo survival in murine blastocysts. Journal of Biological Chemistry 281 6010-6019.

Rossini AA, Appel MC, Williams RM \& Like AA 1977 Genetic influence of the streptozotocin-induced insulitis and hyperglycemia. Diabetes $\mathbf{2 6}$ 916-920.

Sakkas D, Urner F, Menezo Y \& Leppens G 1993 Effects of glucose and fructose on fertilization, cleavage, and viability of mouse embryos in vitro. Biology of Reproduction 49 1288-1292.

Sanguinetti RE, Ogawa K, Kurohmaru M \& Hayashi Y 1995 Ultrastructural changes in mouse Leydig cells after streptozotocin administration. Experimental Animals 44 71-73.

Scarano WR, Messias AG, Oliva SU, Klinefelter GR \& Kempinas WG 2006 Sexual behaviour, sperm quantity and quality after short-term streptozotocin-induced hyperglycaemia in rats. International Journal of Andrology 29 482-488.

Schurmann A, Axer H, Scheepers A, Doege H \& Joost HG 2002 The glucose transport facilitator SLC2A8 is predominantly associated with the acrosomal region of mature spermatozoa. Cell and Tissue Research 307 237-242.

Seethalakshmi L, Menon M \& Diamond D 1987 The effect of streptozotocin-induced diabetes on the neuroendocrine-male reproductive tract axis of the adult rat. Journal of Urology 138 190-194.

Sexton WJ \& Jarow JP 1997 Effect of diabetes mellitus upon male reproductive function. Urology 49 508-513.

Shrivastav P, Swann J, Jeremy JY, Thompson C, Shaw RW \& Dandona P 1989 Sperm function and structure and seminal plasma prostanoid concentrations in men with IDDM. Diabetes Care 12 742-744.

Silvestroni L, Modesti A \& Sartori C 1992 Insulin-sperm interaction: effects on plasma membrane and binding to acrosome. Archives of Andrology 28 201-211.

Tesarik J 2005 Paternal effects on cell division in the human preimplantation embryo. Reproductive Biomedicine Online 10 370-375.

Urner F \& Sakkas D 1996 Glucose participates in sperm-oocyte fusion in the mouse. Biology of Reproduction 55 917-922.

Wang J, Takeuchi T, Tanaka S, Kubo SK, Kayo T, Lu D, Takata K, Koizumi A \& Izumi T 1999 A mutation in the insulin 2 gene induces diabetes with severe pancreatic beta-cell dysfunction in the Mody mouse. Journal of Clinical Investigation 103 27-37.

Wiernsperger NF 2003 Oxidative stress as a therapeutic target in diabetes: revisiting the controversy. Diabetes \& Metabolism 29 579-585.

Wood IS \& Trayhurn P 2003 Glucose transporters (GLUT and SGLT): expanded families of sugar transport proteins. British Journal of Nutrition 89 3-9.

Received 17 April 2008

First decision 27 May 2008

Accepted 12 June 2008 